\title{
Advancing Canadian Urological Association guidelines using GRADE
}

\author{
Philipp Dahm, MD, MD, MHSc, FACS 1,2; Jane Lewis, MD'; Shahnaz Sultan, MD ${ }^{1,3}$ \\ 'Minneapolis VA Medical Center; ${ }^{2}$ University of Minnesota, Department of Urology; ${ }^{3}$ University of Minnesota, Department of Medicine, Division of Gastroenterology; Minneapolis, MN, United States
}

Cite as: Can Urol Assoc J 2018;12(4):93-4. htrp://dx.doi.org/10.5489/cuai.5270

See related guideline on page 85

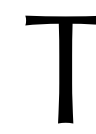

he arena of clinical practice guidelines has undergone a tremendous evolution over the last 10 years. This is largely based on the increasing recognition that past guidelines have been limited by a number of issues, most importantly, the lack of a formal transparent framework for moving from evidence to recommendations. ${ }^{1}$ As a result, the Institute of Medicine (IOM), (now renamed the Health and Medicine Division of the National Academy of Sciences, Engineering and Medicine) published its report, "Clinical practice guidelines we can trust," summarizing key criteria for guidelines to be considered "evidence-based." ${ }^{2}$ These standards have subsequently been adopted by the National Guidelines Clearing House $(\mathrm{NGCH})$ as a resource for guideline documents that meet minimal methodological standards. ${ }^{3}$ This has placed considerable pressure on professional organizations, including those in urology, to raise their methodological standards.

The Canadian Urological Association (CUA) deserves credit for recognizing the changing tides in clinical practice guidelines and making a commitment to the future use of GRADE. GRADE refers to an approach to rating the quality evidence and moving from evidence to decisions that has been adopted by over 100 organizations worldwide, including well-known entities as Cochrane Canada, Cancer Care Ontario, and the Canadian Agency for Drugs and Technology in Health (CADTH). ${ }^{4}$ In urology, the arguably two most influential organizations, the American Urological

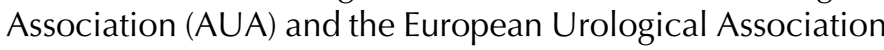
(EAU) are both moving towards GRADE. The AUA so far uses GRADE to rate the quality evidence, whereas the EAU has recently published a full guidance document on perioperative venous thromboembolism prophylaxis that represents the most rigorous urological guideline to date. ${ }^{5}$ The CUA will, therefore, find itself in excellent company, with expanded horizons for collaboration. ${ }^{6}$
The recent CUA guidance on the diagnostic workup and management of antenatally detected hydronephrosis predates the adoption of GRADE and is useful in highlighting both the challenges and opportunities in guideline development. ${ }^{7}$ This particular guideline faces the challenges of tackling a very broad clinical topic that includes both questions of diagnostic accuracy (in a setting where there is no wellestablished reference standard) and therapeutic effectiveness (in an arena marked by a continued paucity of randomized, controlled trials) making this a difficult endeavor irrespective of which methodological approach is used. ${ }^{8}$ Several aspects, though, would be improved upon in a future update based on the use of GRADE.

First, the current guideline was developed using the Oxford Centre of Evidence-Based Medicine framework for rating the quality of evidence, which is limited by its overly strong focus on study design and relative disregard of other domains that affect our confidence in a body of evidence. ${ }^{9}$ This limitation is one that likely resonates with many urologists; we are all aware of examples of studies heralded as "level I evidence," which clearly do not provide high-quality evidence for reasons that may include their inclusion/exclusion criteria, sample size and event rate, or inconsistency when interpreted in the context of other relevant studies that make up the body of relevant evidence. Second, the use of the International Consultation in Urology framework for developing recommendations does not meet current IOM or NGCH standards. ${ }^{10}$ Specific shortcomings are related to the incomplete separation of the quality of evidence and strength of recommendations, as well as the lack of explicit criteria that would allow the user to determine how the guideline panel arrived at a given recommendation.

Future CUA guidelines using the GRADE evidence-todecision framework would explicitly document the panel members' deliberations about core domains, such as the presumed magnitude of the potential benefits and harms and their respective ratio, underlying assumptions about target populations' values and preferences, as well as resource requirements. ${ }^{1112}$ When relevant, panelists would also have an opportunity to formally consider issues sur- 
rounding acceptability, feasibility, and equity of the recommended clinical action. Lastly, recommendations would be consistently worded as "the panel recommends" for strong recommendations vs. "the panel suggests" for conditional or weak recommendations.

For example, a future update of this guideline may read, "In infants with an antenally detected anterior-posterior renal pelvis diameter of $\geq 7 \mathrm{~mm}$, the panel recommends a renal ultrasound (strong recommendation based on low quality evidence)." Such a strong recommendation may be appropriate even in absence of high-quality evidence if the benefit clearly outweighed potential harms of the interventions (which in this case may include the risk of overdiagnosis and overtreatment). This example highlights how the certainty in the evidence does not alone determine the strength of a recommendation and how strong recommendations (under specific circumstances) are possible even in the presence of lower-quality evidence. Based on GRADE, this would mean that performing a renal ultrasound would be appropriate in the vast majority of infants (but not necessarily all) and that parents should have an expectation that this service be provided. ${ }^{13}$ Some strong recommendations may also lend themselves as performance measures.

In contrast, a weak recommendation may read, "The panel suggests continuous antibiotic prophylaxis in infants with confirmed Grade 3 or 4 hydronephrosis (weak recommendation based on low-quality evidence)." Such a recommendation would reflect not only the lower-quality evidence, but also potentially an underlying uncertainty about how infants' parents may value the potential benefits of continuous antibiotic prophylaxis (less urinary tract infections, potentially reduced risk of clinically relevant renal damage) vs. its potential harms (inconvenience and costs of daily antibiotic intake; drug-related adverse effects, and development of antibiotic resistance). A weak recommendation according to GRADE would signal that while continuous antibiotic prophylaxis may be appropriate in many patients, shared decision-making with the parents is critical before embarking on a long-term management pathway.
Adoption of GRADE can, therefore, be expected to enhance the transparency and accountability of the CUA's guidelines, and ultimately improve patient care.

Competing interests: The author reports no competing personal or financial interests related to this work.

\section{References}

1. Gupta $M$, McCauley J, Farkas A, et al. Clinical practice guidelines on prostate cancer: A critical appraisal. J Urol 2014;193:1153-8. https://doi.org/10.1016/i.juro.2014.10.105

2. Institute of Medicine (U.S.). Committee on Standards for Developing Trustworthy Clinical Practice Guidelines. Graham R. Clinical practice guidelines we can trust. Washington, DC: National Academies Press 2011.

3. National Guideline Clearinghouse: Agency for Healthcare Research and Quality (AHRQ); 2008 [updated 07/21/2008]. Available at www.guideline.gov. Accessed Feb. 26, 2018.

4. GRADE Working Group. Available at http://www.gradeworkinggroup.org. Accessed Feb. 22, 2018.

5. Tikkinen KA, Cartwright R, Gould MK, et al. European Urological Association guideline on thromboprophylaxis: European Urological Association; 2017. Available at http://uroweb.org/individual-guidelines/ non-oncology-guidelines/. Accessed Feb. 25, 2018.

6. Dahm P, Chapple CR, Konety BR, et al. The future of clinical practice guidelines in urology. Eur Urol 2011;60:72-4. https://doi.org/10.1016/i.eururo.2011.04.007

7. Capolichio JP, Braga LH, Szymanski KM. Canadian Urological Association/Pediatric Urologists of Canada guidelines on the investigation and management of antenatally detected hydronephrosis. Can Urol Assoc J 2017 Dec. 22; Epub ahead of print. https://doi.org/10.5489/cuaj.5094

8. Welk B, Afshar K, MacNeily AE. Randomized controlled trials in pediatric urology: Room for improvement. J Urol 2006;176:306-9. https://doi.org/10.1016/S0022-5347(06)00560-X

9. Singh JC, Dahm P. Evidence-based urology in practice: What are levels of evidence? BJU Int 2009; 103:8601. https://doi.org/10.1111/i.1464-410X.2009.08429.x

10. Abrams $\mathrm{P}$, Khoury $\mathrm{S}$. International Consultation on Urological Diseases: Evidence-based medicine overview of the main steps for developing and grading guideline recommendations. Neurourol Urodyn 2010;29:116-8. https://doi.org/10.1002/nau.20845

11. Alonso-Coello P, Oxman AD, Moberg J, et al. GRADE Evidence to Decision (EtD) frameworks: A systematic and transparent approach to making well informed healthcare choices. 2: Clinical practice guidelines. BMJ 2016;353:i2089. htrps://doi.org/10.1136/bmi.i2089

12. Alonso-Coello P, Schunemann HJ, Moberg J, et al. GRADE Evidence to Decision (EtD) frameworks: A systematic and transparent approach to making well-informed healthcare choices. 1: Introduction. BMJ 2016;353:i2016. htrps://doi.org/10.1136/bmi.i2016

13. Canfield SE, Dahm P. Rating the quality of evidence and the strength of recommendations using GRADE. World J Urol 2011;29:311-7. https://doi.org/10.1007/s00345-011-0667-2

Correspondence: Dr. Philipp Dahm, Minneapolis VA Health Care System, Minneapolis, MN, United States; pdahm@umn.edu 